après révision — qu'elle présente de points forts, à savoir : le fait de disposer de trois états du texte, d'avoir des copies indépendantes et de bénéficier ainsi d'une grande complémentarité des états du texte.

En dernier lieu, l'éditrice se propose d'étudier de plus près les caractéristiques juridique, historique et autobiographique de la Vita, en insistant d'abord sur la dimension « jurislittéraire » (125), occultée jusqu'ici au profit de la seule prise en considération du caractère autobiographique. Un deuxième modèle apparaît, celui des historiens : César, Plutarque, mais aussi Suétone et Tacite. Les Vies de saints constituent un troisième modèle, attestant ainsi la richesse d'un texte convoquant toute une palette de savoirs historiques, théologiques littéraires, médicaux et juridiques en vue d'éclaircir et de défendre la somme qu'est l'Histoire.

Au terme d'un parcours inaugural livrant au lecteur toutes les informations nécessaires à la bonne compréhension de la Vita, sans qu'à aucun moment les questions en suspens ne soient occultées, le lecteur peut goûter enfin un texte jusqu'ici difficilement accessible, et ce dans une édition qui satisfait désormais pleinement aux exigences universitaires. La mise en page du texte est claire, et la traduction en miroir, précise et élégante, comme l'atteste, par exemple, celle du poème que J.-A. de Thou adresse « Au très illustre Prince Charles, cardinal de Vendôme » (576-585). Si l'on peut regretter l'absence d'une bibliographie, sinon systématique, du moins susceptible de rendre service à l'amateur éclairé comme au jeune chercheur perdu dans le dédale des références, force est d'admettre que l'établissement d'un appareil de notes précises et copieuses ainsi que les renvois à des ouvrages offrant une bibliographie thuanienne importante et récente (14) viennent finalement combler nos attentes. Nul doute, cette édition de la Vita fait date.

Thierry victoria, Classes Préparatoires aux Grandes Écoles, Poitiers ; CEMar, Université de Picardie-Jules Verne.

\title{
Patronnes et mécènes en France à la Renaissance
}

Études réunies et présentées par Kathleen Wilson-Chevalier avec la collaboration d'Eugénie Pascal.

St-Étienne : Publications de l'Université de Saint-Etienne, L'École du genre, 2007, $682 \mathrm{p}$.

Vingt-cinq seiziémistes ont contribué à ce volume qui met en lumière les femmes, auteurs ou non, commanditaires ou protectrices d'auteurs et d'artistes sinon 
« bâtisseurs » qui ont affirmé, sciemment ou non, leur pouvoir de modifier ou de transformer les traditions établies, voire d'en créer de nouvelles.

L'introduction de Wilson-Chevalier (pp.7-42) indique qu'elle incorpore dans la première partie du volume (pp. 45-131) la biographie de plusieurs femmes fortes et un survol du mécénat féminin d'œuvres écrites (manuscrites ou imprimées) tant dans le cadre social et politique que religieux. Chaque étude de la seconde partie (pp.135-576) sera dédiée à une femme de la haute noblesse en raison du rôle qu'elle a joué pendant sa vie mais aussi pour les conséquences de ce qu'elle a accompli, tant dans les mœurs, que dans les arts et l'histoire. On y trouvera des portraits, des planches, des reproductions photographiques de châteaux, cloîtres, tombeaux, bas-reliefs, monuments funéraires et sculptures, d'illustrations de manuscrits et de livres imprimés, minutieusement répertoriés (pp. 669-678).

Selon Wilson-Chevalier, la seconde partie confirme la prémisse de la première, c'est-à-dire que certains hommes de la Renaissance étaient profondément conscients de l'importance de la femme mécène. Elle souligne enfin que le but de ce volume collectif sur les patronnes et mécènes est de démontrer que l'insertion des femmes dans l'Histoire est bien (trop) souvent l'œuvre de leur propre volonté.

Susan Broomhall note dans «Au-delà de la Cour: patronnes et mécènes du manuscrit à l'imprimé » (pp. 45-58) que les bibliothèques de femmes nobles contenaient de riches collections de manuscrits; elle aussi souligne une prolifération de bibliothèques et de collections et le rôle que les femmes (qui n'appartenaient pas toutes aux milieux de la cour) y ont joué après l'apparition de l'imprimerie. La plupart des œuvres destinées et souvent dédiées à un public féminin leur proposaient un modèle de comportement. Les patronnes désireuses de forger des modèles positifs à l'intention de leur sexe ont donc offert un soutien financier et social à des textes plus conformes à leur penchant intellectuel ou personnel.

Aurore Evain divise « Les reines et princesses de France, mécènes, patronnes et protectrices du théâtre au XVI ${ }^{\mathrm{e}}$ siècle $\gg$ (pp. 59-99) en plusieurs sections : 1400-1530 les précurseuses, Le patronage humaniste de Marguerite de Navarre, Les protections littéraires de Marguerite de France, duchesse de Berry et de Savoie (1523-1574), Le mécénat politique de Catherine de Médicis (1519-1589), Le patronage religieux de Jeanne d'Albret (1528-1572) et Les héritières, ce dernier touchant (logiquement) plus au XVII ${ }^{\mathrm{e}}$ siècle qu'au XVI ${ }^{\mathrm{e}}$, et divisé en quatre sous-titres. Cette étude, riche de notes et de citations, sera une source de recherche utile pour tout historien du théâtre.

Dans « Princesses épistolières au tournant du XVI ${ }^{e}$ au XVII ${ }^{e}$ siècle: Consommatrices de culture, mécènes et/ou propagandistes ? » - titre provocateur s'il en est (pp.101-131) —, Eugénie Pascal répartit une quinzaine d'épistolières, 
de Catherine de Bourbon à Marguerite, reine de France, en deux camps, les protestantes et les catholiques. Son étude révèle qu'il est très peu question d'art, de musique, de divertissements ou de belles-lettres dans les lettres de ces princesses. Toute production culturelle y est abordée pour sa valeur politique, morale ou religieuse. On ne parle chiffons que dans le but de savoir ce qui se porte d'une cour à l'autre afin d'affirmer son rang et son prestige. Pour le mobilier, la décoration des châteaux, surtout celle des jardins, et le luxe des équipages, il s'agit de statut plus que d'esthétique. En peinture, cependant, les portraits sont indispensables pour se faire connaître et surtout reconnaître. Le théâtre est loué si les acteurs sont bons et si le sujet n'est pas choquant. Elles lisent mais disent bien peu sur les livres qu'elles s'envoient. Ce sont les arts oratoires et les sermons qui semblent importants pour ces dames, et bien qu'elles aient toutes encouragé les arts ou même écrit des vers, Pascal note que la polémique est fréquente dans leurs lettres, qui se réfèrent souvent aux discours politiques, révélant ainsi leur fonction pragmatique en vue de protéger et surtout de promouvoir leur famille ou leur coterie.

Passons maintenant à la seconde partie. Anne de France est le sujet de deux études. Dans « Sainte Anne et son mécénat » (pp. 135-154), Elizabeth L’Estrange démontre que pour les châteaux et chapelles qu'elle fait construire ou décorer, la princesse a recours aux thèmes associés à Sainte Anne (l'immaculée conception, la sagesse, l'éducation de la Vierge). Elle affirme ainsi son pouvoir acquis en tant que régente et son rôle d'éducatrice de sa fille Suzanne. Elodie Lequain souligne dans « Anne de France et les livres: la tradition et le pouvoir » (pp. 155-168) que la princesse, maillon prestigieux de l'héritage féminin du savoir, a enrichi la superbe collection de manuscrits qu'elle avait hérité de ses ancêtres avec des œuvres de son choix, d'autres reçues en hommage à son autorité politique, et d'autres de sujets variés - poésie, médecine, histoire, dévotion — écrites pour elle par des auteurs qui connaissaient ses goûts.

Le mécénat d'Anne de Bretagne est le sujet de deux études. L’Estrange (pp.169193) montre que la duchesse affirme simultanément son pouvoir sur la Bretagne et dans les territoires dont elle est reine. Elle analyse une sélection de manuscrits enluminés et de tombeaux commandés par la reine qui, comme Anne de France, invoque Sainte Anne, mère choisie par Dieu et qui, en l'absence d'un héritier mâle, protège ses deux filles. Cynthia Brown (pp.195-224) analyse les œuvres qui révèlent le pouvoir de la reine sur les hommes qui ont écrit ou traduit pour elle, mais aussi sur ceux qui ont publié et illustré celles qu'on lui offrait. Elle découvre qu'en dépit de leurs dédicaces dithyrambiques, certaines images littéraires et des miniatures cachaient des allusions négatives quant au pouvoir des femmes. 
Jens Ludwig Burk développe et confirme son thème «A 'L'anticque' à Nantes et 'A la moderne' à Brou : styles architecturaux et conception de la statuaire funéraire au moment du passage du gothique tardif à la Renaissance $\gg$ (pp. 225-250) à l'aide de nombreuses reproductions d'œuvres funéraires commandées par Anne de Bretagne et Marguerite d'Autriche à des moments marquant la fin d'une période de leur vie. Selon Burk, bien qu'Anne ait choisi le style de la Renaissance et Marguerite celui du gothique tardif, les gisants de Nantes sont plus traditionnels que ceux de Brou, dont les effigies funéraires de la représentation vivante imposent une vision stylistique plus novatrice que celle de la reine Anne.

« Louise de Savoie, ses enfants et ses livres : Du pouvoir familial au pouvoir d'Etat » (pp. 251-281) de Mary Beth Winn souligne que le renom de la comtesse d'Angoulême est dû en partie au rôle qu'elle a choisi dans l'éducation de ses enfants, et surtout de son fils bien avant qu'il n'accède au trône. Lors de son mariage, la bibliothèque de Cognac comptait une superbe collection de manuscrits qu'elle a enrichie, et elle s'est entourée d'artistes et d'auteurs érudits. Elle commande ou on lui dédie des œuvres de morale, de religion, de philosophie, et d'histoire. Winn reproduit un inventaire détaillé de livres lui ayant appartenu, de manuscrits et de livres imprimés qu'elle a commandés, et d'autres ayant pu lui appartenir.

Michel Melot répond à une question souvent posée: la construction, l'embellissement et le trésor des monastères féminins relevaient-ils du mécénat aristocratique ? Les documents d'archives qu'il cite et les œuvres d'art reproduites dans « Les Bâtisseuses de Fontevraux $\gg$ (pp. 283-302) prouvent que la richesse des objets d'art apportés en dot par les religieuses aristocrates faisaient de Fontevraux, la plus riche des abbayes de femmes en France, et un véritable palais princier, dans les limites du respect de la règle monastique.

Marguerite de Navarre fait l'objet de deux études, l'une, « La protection de vostre faveur $\gg$, de Barbara Stephenson, sur son patronage humaniste (pp.303-319) et l'autre d'Anne Funke, sur son « Influence spirituelle sur les vitraux de la cathédrale Sainte-Marie d'Auch » (pp. 321-340). Stephenson indique que Marguerite protégea un si grand nombre d'auteurs humanistes et de réformateurs religieux que certains se contentaient d'être associés à son nom sans chercher à se procurer une récompense matérielle. Elle souligne son patronage des institutions éducatives en France, mettant ainsi en lumière l'importance de son rôle dans la culture de l'Europe pré-moderne. Funke décrit ou reproduit les vitraux ajoutés vers ou après 1530 à ceux de la cathédrale d'Auch, dont Marguerite devint chanoinesse en tant qu'épouse du roi Henri II de Navarre. Elle lit dans ces vitraux le rayonnement spirituel et littéraire 
de la reine de Navarre et surtout la promotion de la puissance des Valois, accentuée par les lys monumentaux de pierre et de verre du chœur.

Dans « L'épreuve du mécénat: Alienor d'Austriche, une reine de France effacée ? » (pp. 341-380), Annemarie Jordan et Kathleen Wilson-Chevalier suggèrent que qu'Aliénor, bien que la moins visible des sœurs de Charles-Quint, a su affirmer comme princesse, puis reine du Portugal, puis seconde épouse de François $\mathrm{I}^{\mathrm{er}}$, son individualité en tant que mécène cultivée. Elles ajoutent qu'elle a souvent mis la littérature et les beaux-arts au service de la politique pour favoriser ses causes personnelles.

Le but de Sigrid Ruby est de démystifier l'image populaire et misogyne de «Diane de Poitiers : veuve et favorite » (pp.381-399), de séductrice belle et cupide. La loyauté de son mari Louis de Brézé envers la couronne l'aide à promouvoir sa position privilégiée en tant que maîtresse du Dauphin, et lors de son veuvage, elle fera construire de superbes monuments à sa mémoire. Après l'accession au trône d'Henri II, favorite et confidente du roi, elle jouera à la cour un rôle de premier plan. Ce sont cependant sa haute naissance et sa fortune qui lui ont permis de commanditer de luxueuses demeures, des œuvres d'art, de collectionner des œuvres antiques, et de s'attacher les services de poètes cotés.

Antoinette de Bourbon est le sujet de deux études. Le titre choisi par Jessica Munns et Penny Richards, « Antoinette de Bourbon, première duchesse de Guise, et Rémy Belleau: construction d'un tombeau, création d'un mythe » (pp. 401-417), annonce le thème de leur étude. La maison de Lorraine s'étant attribué le droit de revendiquer la couronne de Jérusalem, la piété religieuse et la gloire familiale étaient la raison d'être des projets commandités par la duchesse. Ainsi que le montre l'un des dessins originels du tombeau du premier duc de Guise, œuvre du Primatice, Claude de Guise, un sceptre à la main, semble être assis sur un trône. La duchesse exalte ainsi la conviction des Guises qu'ils étaient de souche royale et guerriers de Dieu. R. Belleau, poète attitré des Guises, ne manquera pas d'affirmer ce mythe en décrivant dans La Bergerie le tombeau de ce grand prince. « Le triomphe d'une mater familias » (pp. 419-431) de Camille Grand-Dewyse se penche sur une plaque émaillée commandée par le cardinal Charles de Lorraine à Léonard Limosin représentant sa mère, Antoinette de Bourbon, entourée des hommes de sa famille. On la voit comme une Vénus chrétienne luttant contre l'hérésie, mais guidant ses fils vers une politique dans laquelle la violence cède la place à une attitude plus conciliante en vue d'une solution pacifique. Cette plaque révèle l'influence de cette mère chef de famille et l'importance du rôle que les femmes conscientes de leur pouvoir jouaient dans la société. 
Dans « Le mécénat meurtrier, l'iconoclasme et les limites de l'acceptable: Anne d'Este, Catherine-Marie de Lorraine et l'anéantissement d'Henri III » (pp. 433-454), Dora Polachek souligne que l'art et la propagande allaient de pair dans le mécénat des Guises et examine le rôle de ces deux femmes dans la métamorphose d'une affaire de famille en affaire d'État, dont les conséquences seront l'avilissement et la mort du roi. L'attitude, les faits et gestes d'Anne d'Este et de sa fille lors de l'assassinat du duc et du cardinal de Guise révèlent un spectacle soigneusement orchestré, mais dans lequel le comportement d'Anne restera dans la limite de ce qu'on accepte d'une femme de pouvoir, alors que sa fille passera outre la barrière des sexes en assumant un rôle masculin. Les larmes de l'une et les cris d'indignation de l'autre, arme à double tranchant, ont néanmoins anéanti la figure politique du roi et précipité la fin du règne des Valois.

La part du lion revient à Catherine de Médicis, à qui les sept dernières contributions (pp. 455-576) sont consacrées. La reine mécène idéale de la Renaissance : «Catherine de Médicis définie par elle-même ou par les autres », de Sheila Ffolliott, analyse un livre de Nicolas Houël dédié à la reine alors âgée de soixante-sept ans, dont il cherchait le patronage, et décrivant le mécénat idéal féminin: piété, charité et dévotion à la mémoire de leur royal époux, construction d'établissements religieux et soutien d'institutions charitables. Ffolliott démontre aisément que Catherine a largement outrepassé les limites du rôle décrit par Houël pour les femmes mécènes, tant à la cour que dans le royaume.

Caroline zum Kolk étudie son mécénat d'après sa correspondance et indique que, sur un total de plus de six mille lettres, peu sont relatives au mécénat. Plus de quatre-ving lettres (1529-1574) fournissent bon nombre d'indices quant à la diversité de son engagement culturel. Outre l'organisation de cérémonies, les beaux-arts, l'architecture, l'orfèvrerie et les étoffes qui révèlent ses goûts personnels, ces lettres confirment qu'elle entretenait des relations épistolaires célébrant la royauté et consacrant des liens de parenté qui lui permettaient d'exercer son influence dans le domaine culturel [et nous ajouterions : politique] dans un mécénat marqué par un grand dynamisme.

Dans « Les coulisses du pouvoir: châteaux, jardins et fêtes, quelques aspects du mécénat (transgressif) de Catherine de Médicis $\gg$, Laurent Odde la décrit comme l'une des femmes politiques les plus importantes de son temps mais aussi l'une des mécènes les plus singulières de la période moderne. Sa passion pour l'architecture la pousse à faire construire ou reconstruire de nombreuses résidences. Elle fera de ses demeures et de leurs superbes jardins le cadre idéal de rencontres politiques et 
de festivités aux décors fabuleux, et on ne trouvera l'écho de ce goût de la mise en scène du pouvoir royal que sous le règne de Louis XIV.

C'est la résidence parisienne de la reine, détruite au XVIII ${ }^{\mathrm{e}}$ siècle, qu'étudie Chantal Turbide dans « Catherine de Médicis, mécène d'art contemporain : l'hôtel de la Reine et ses collections ». Àl'aide de sources manuscrites et iconographiques, elle reproduit un plan des appartements que la reine avait aménagés, meublés et décorés. L'inventaire après son décès (outre les œuvres peintes sur les murs et les plafonds, boiseries, parquets, plus de mille objets seuls ou de lots sont répertoriés) confirme que les collections d'art de la reine mère, comme les festivités, étaient intimement liées au rôle joué par sa résidence dans l'affirmation du pouvoir de la monarchie française.

Dans « Catherine de Médicis et les portraitistes français », Alexandra Zvereva prouve que loin d'atterrer les portraitistes de son époque, ainsi qu'une biographie l'a décrite, la reine était passionnée des portraits à la française de Jean et François Clouet dont elle possédait plusieurs centaines d'œuvres. De plus, après 1559, elle avait aussi ses propres peintres, et 482 peintures seront recensées après sa mort dans sa résidence parisienne. Protectrice et mécène des peintres royaux, elle leur offrait non seulement des gages généreux mais aussi une reconnaissance, une dignité et une liberté jusque-là inaccessible aux personnes de leur condition, transformant l'ouvrier en artiste.

La reine appréciait la peinture, mais sa prédilection pour les bijoux est bien connue. Dans « Catherine de Médicis et ses orfèvres : autorité, précision, exigence », Michèle Bimbenet-Privat indique que les orfèvres nommés parmi ses fournisseurs ou subventionnés par sa Maison étaient tous parisiens. Elle leur communiquait des directives quant au dessin ou à la devise qu'elle avait composée et de minutieuses descriptions, parfois accompagnées de croquis de sa propre main, personnifiant ainsi chaque bijou ou objet d'orfèvrerie qu'elle commandait pour les membres de sa famille ou ceux de sa maison, révélant aussi sa grande sagesse dans les cas de présents diplomatiques.

L'influence des mécènes ne cesse pas à leur mort, et Kerrie-rue Michahelles le prouve dans «Apprentissage du mécénat et transmission matrilinéaire du pouvoir. Les enseignements de Catherine de Médicis à sa petite-fille Christine de Lorraine ». Elle y interprète le testament de la reine, qui souhaitait maintenir la lignée des Valois sur le trône de France. La première et plus importante légataire est Christine de Lorraine, qu'elle avait élevée ; suivent Louise de Lorraine-Vaudémont, son petitfils Charles de Valois (fils bâtard de Charles IX) et son fils Henri III. Anticipant le mariage de Christine de Lorraine avec Ferdinand $\mathrm{I}^{\mathrm{er}}$ de Médicis, Catherine fait de 
sa petite-fille le pivot de son projet politique à long terme, assure le double lignage de Christine et lui transmet l'hôtel de la reine, ses propriétés italiennes et 200.000 écus-pistoles que lui doit le grand-duc de Toscane. C'est donc à la future grandeduchesse de Toscane qu'elle confie ses propriétés et maintient la lignée des Valois sur le trône de France par la mise en place d'une alliance politique entre la France et les Médicis.

Notices sur les contributrices et contributeurs et un résumé des articles (pp. 577-594), la bibliographie (pp. 595-640, l'Index nominum (pp. 641-667), la table des illustrations et planches (pp.669-678) et la table des matières complètent le volume

La traduction étant souvent un labeur subjectif, nous avons souvent regretté l'absence (obligatoire et logique) du texte original anglais de plusieurs articles, mais nous ne pouvons que féliciter les contributeurs et les éditrices de ce remarquable volume. La qualité de la recherche dans ce vaste sujet, jusqu'à présent peu exploré, fait de cet outil de travail une mine d'or pour la génération « montante » de seiziémistes.

Finalement, il nous faut applaudir la SIEFAR qui, depuis sa création, poursuit avec le plus grand succès sa mission de faire connaître et reconnaître les femmes auteurs de l'Ancien Régime et les œuvres qu'elles ont créées. Il semble donc indispensable de citer le rôle capital de notre collègue Eliane Viennot, maître d'œuvre de ce projet qui n'a cessé de croître, dont l'impeccable réputation n'est plus à faire.

RÉGINe Reynolds-CORnell, Agnes Scott College

\section{Jean Thenaud}

\section{Traicté de la Cabale}

Édition établie et annotée par Ian Christie Miller avec la collaboration de François Roudaut et la participation de Claire César, Pierre Gauthier et Jean Timotéi Paris : Honoré Champion, 2007, 496 p.

Le cordelier Jean Thenaud (fin XVe siècle, c.1542), protégé de Louise de Savoie et abbé de Melinais dans le diocèse d'Angers, est l'un des premiers représentants français de cette kabbale chrétienne dont on sait qu'elle trouva à s'illustrer, à la fin du XV et au début du XVI ${ }^{\mathrm{e}}$ siècle, dans les œuvres de Paul Rici, Jean Reuchlin et Jean Pic de la Mirandole. On doit à François Secret d'avoir autrefois mieux fait connaître dans un court article («Jean Thénaud, voyageur et kabbaliste de la Renaissance », Bibliotheque d'Humanisme et Renaissance XVI (1954) p. 139-144.) cette figure ori- 